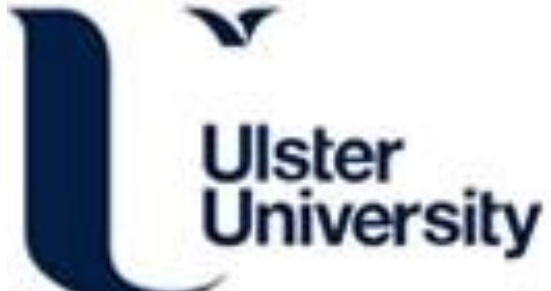

\section{Comparison Analysis: Single and Multichannel EMD-Based Filtering with Application to $\mathrm{BCl}$}

Gaur, P., Kaushik, G., Pachori, R. B., Wang, H., \& Prasad, G. (2018). Comparison Analysis: Single and Multichannel EMD-Based Filtering with Application to BCI. In M. Tanveer, \& R. B. Pachori (Eds.), Machine Intelligence and Signal Analysis (Vol. 748, pp. 107-118). (Advances in Intelligent Systems and Computing). Springer Singapore.

Link to publication record in Ulster University Research Portal

\section{Published in:}

Machine Intelligence and Signal Analysis

Publication Status:

Published (in print/issue): 08/08/2018

\section{Document Version \\ Author Accepted version}

\section{General rights}

Copyright for the publications made accessible via Ulster University's Research Portal is retained by the author(s) and / or other copyright owners and it is a condition of accessing these publications that users recognise and abide by the legal requirements associated with these rights.

\section{Take down policy}

The Research Portal is Ulster University's institutional repository that provides access to Ulster's research outputs. Every effort has been made to ensure that content in the Research Portal does not infringe any person's rights, or applicable UK laws. If you discover content in the Research Portal that you believe breaches copyright or violates any law, please contact pure-support@ulster.ac.uk. 


\title{
Comparison analysis: single and multichannel EMD based filtering with application to BCI
}

\author{
P. Gaur, G. Kaushik, R. B. Pachori, H. Wang, and G. Prasad
}

\begin{abstract}
A brain-computer interface (BCI) aims to facilitate a new communication path that translates the motion intentions of a human into control commands using brain signals such as magnetoencephalography (MEG) and electroencephalogram (EEG). In this work, a comparison of features obtained using single channel and multichannel empirical mode decomposition (EMD) based filtering is done to classify the multi-direction wrist movements based MEG signals for enhancing a brain-computer interface (BCI). These MEG signals are presented as a dataset 3 as part of the BCI competition IV. These single channel and multichannel EMD methods decompose MEG signals into a group of intrinsic mode functions (IMFs). The mean frequency measure of these IMFs has been used to combine these IMFs to obtain enhanced MEG signals which have major contributions from the low-frequency band $(<15 \mathrm{~Hz})$. The shrinkage covariance matrix has been computed as a feature set. These features have been used for the classification of MEG signals into multidirection wrist movements using the Riemannian geometry classification method. Significant improvement of $>8 \%$ in the test stage using the multichannel EMD based filtering and $>4 \%$ when compared with single channel EMD method and BCI competition winner respectively. This analysis offers evidence that the multichannel EMD based filtering has the potential to be used in online BCI systems which facilitate a broad use of noninvasive BCIs.
\end{abstract}

Pramod Gaur

Intelligent Systems Research Centre, Ulster University, Derry, UK., e-mail: p.gaur@ulster.ac.uk

Geetika Kaushik

Intelligent Systems Research Centre, Ulster University, Derry, UK.

Ram Bilas Pachori

Discipline of Electrical Engineering, Indian Institute of Technology Indore, India.

Hui Wang

School of Computing and Mathematics, Ulster University, Jordanstown, UK.

Girijesh Prasad

Intelligent Systems Research Centre, Ulster University, Derry, UK. 


\section{Introduction}

Most people suffering from severe motor disabilities, particularly those who are totally paralysed may need a communication pathway which do not need muscle control. Many studies use brain signals such as magnetoencephalography (MEG) or electroencephalogram (EEG) which serves as a basis for this new communication pathway called a brain-computer interface (BCI) system. It aims to facilitate a new communication path that translates the motion intentions of a human into control commands for an output device using brain signals such as EEG or MEG [22, 3]. However, these electrophysiological signals have low signal-to-noise ratio (SNR) due to external interferences such as electrical power line, etc. and other artefacts resulting from muscle movement, electromyogram (EMG) or eye movements, electrooculogram (EOG) interferences resulting in degraded classification accuracy. It is important to remove these interferences in the preprocessing step to achieve high accuracy in classification problems. To handle this problem, a number of studies have been carried out wherein research groups studied common spatial pattern (CSP) [13] and some extension methods based on the CSP algorithm [1]. Other research groups have utilized the space of symmetric positive definite matrices (SPDM) and computed covariance matrices as a feature set and then further classified using the Riemannian geometry framework [2].

In recent times, empirical mode decomposition (EMD) has shown potential as a very promising decomposition technique to analyse EEG signals [11]. It has been used for the classification of epileptic EEG signals [20,19] and motor imagery BCI classification problems [5]. However, the single channel EMD method has some potential issues such as mode-mixing and frequency localisation problems. To address these issues different variants have been proposed as an extension to single channel EMD method namely, ensemble EMD (EEMD) which adds white Gaussian noise (WGN) [23] but is computationally expensive, and multivariate EMD (MEMD) $[14,16]$ which is a multichannel extension of EMD. These methods adaptively decompose the MEG/EEG signal into a set of intrinsic mode functions (IMFs). Some of these IMFs contain noise and residuals as well. The important challenge is to identify the IMFs which are of interest and the remaining IMFs may be discarded. To address this issue, a single channel EMD based filtering (EMDBF) [5] and a multichannel EMD based filtering (MEMDBF) [8] method have been proposed. These filtering techniques identified the IMFs which are of interest and discarded the rest of them based on a statistical measure namely, mean frequency. Mean frequency computation has been carried out using Fourier spectrum [10]. Then, the identified IMFs are summed to enhance the EEG/MEG signals. These techniques have shown potential to classify the motor imagery EEG signal into two and four classes $[5,8,7]$. In addition, these methods have been studied to classify the MEG signals to address the multi-class classification problem $[6,12,9]$. In this paper, a comparative study of features extracted after applying the EMDBF and MEMDBF algorithms is done to classify MEG signals recorded during hand movements in four directions.

The remaining paper is organised as follows: Section 2 provides the details of data set used for our study and Section 3 discusses the background of EMD and 
MEMD related decomposition techniques respectively. Section 4 presents the analysis of results obtained by comparing the MEMDBF with EMDBF and BCI competition winners. Finally, section 5 describes the conclusions of this analysis.

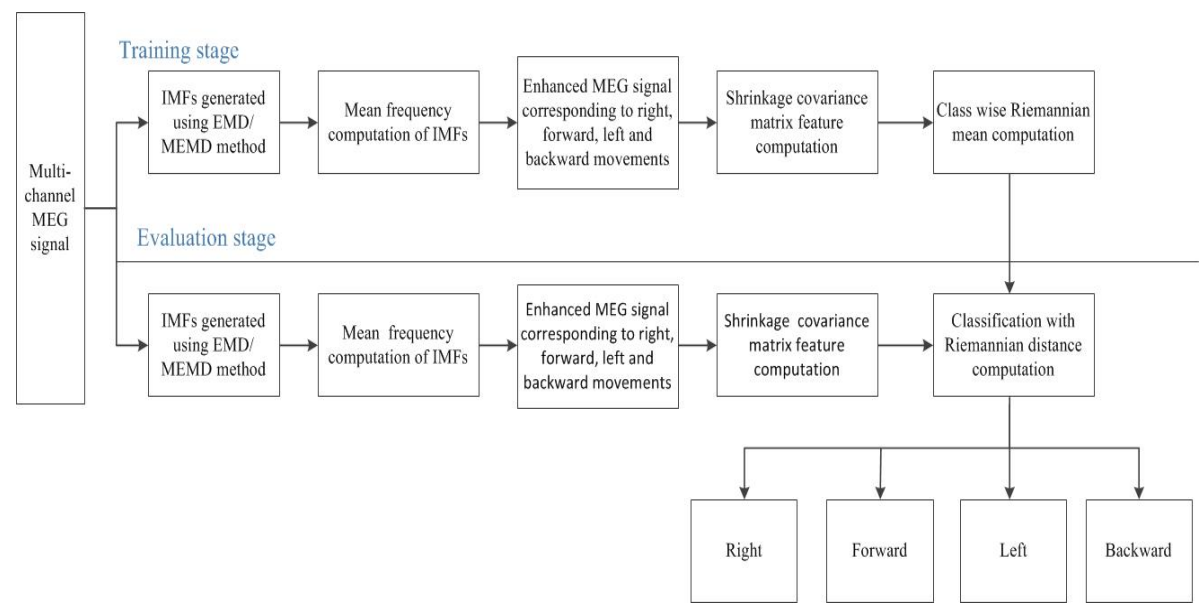

Fig. 1 Block diagram of the proposed methodology

\section{Materials}

The BCI competition IV dataset 3 contains MEG signals for four classes, namely: right, forward, left, and backward movements. These MEG signals from 10 channels above the motor areas have been used for the study as shown in Fig 2. The dataset contains data on two subjects S01 and S02. Each subject contains MEG signals for one training session and one test session. A training set contains 40 trials of each class giving a total of 160 trials for S01 and 160 trials in subject S02 respectively. In evaluation session, there are 74 trials in S01 and 73 trials in S02 to be classified in four different classes [17]. These signals are sampled at $400 \mathrm{~Hz}$. Each trial of the right, forward, left, and backward movements MEG signals contain 400 sample points giving a trial length of $1 \mathrm{sec}$. This dataset has been extensively studied by different research groups in a number of studies [6,9,12,17]. For more details refer to [21]. 


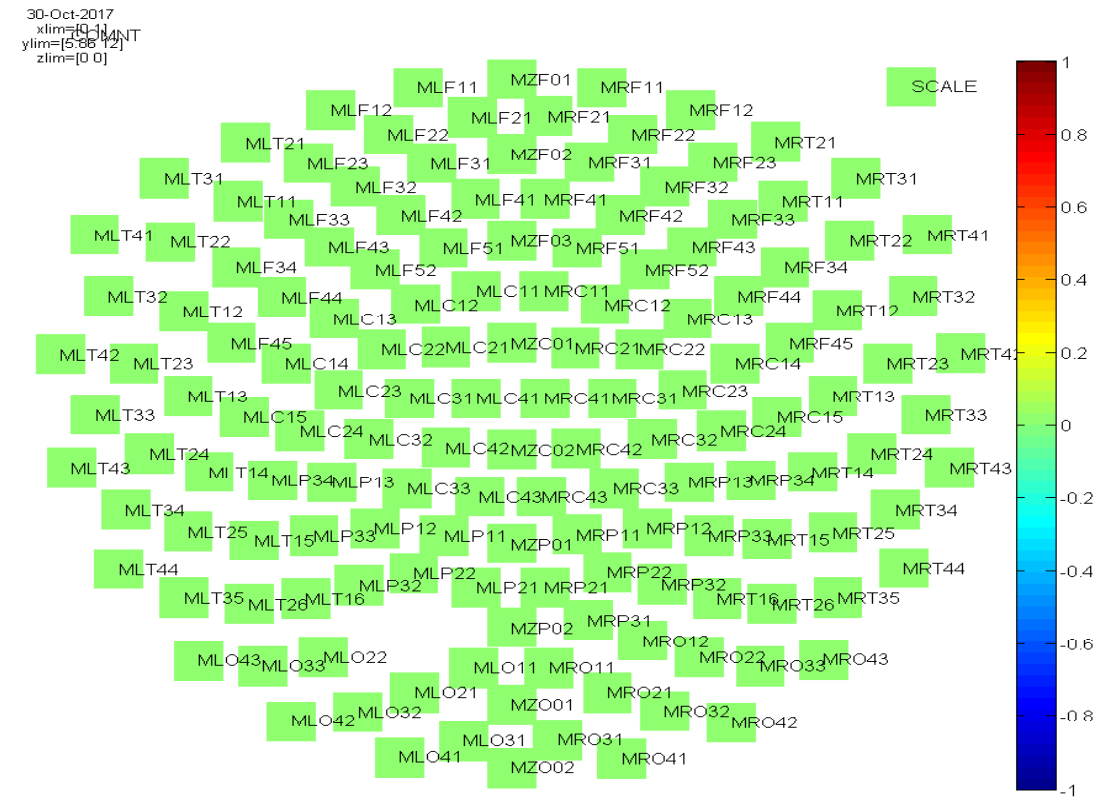

Fig. 2 MLC21, MLC22, MLC23, MLC32, MLC31, MLC41, MLC42, MZC01, MZC02, and MRC41 Channels are used for the present work.

\section{EMD and MEMD algorithm}

This article describes a comparative study between single channel EMD based filtering [5] and MEMD based filtering [8, 7] to classify the multi-direction wrist movements based MEG signals for enhancing a BCI is carried out. The EMD method [11] breaks MEG signals into a set of IMFs. The MEMD method [14] simultaneously decomposes multichannel MEG signals into a set of multivariate IMFs (MIMFs). These IMFs/ MIMFs can be considered narrow-band, amplitude, and frequency modulated (AM-FM) signals. 
The recorded MEG signals are highly non-stationary and non-linear by nature. They also suffer from low SNR. Also, there is a strong possibility that they may contain disturbances from electromyography (EMG), electrooculography (EOG) and power line etc [15]. Therefore, the MEG signals of interest pertaining to a particular cognition task or actual movements may contain high noise which may lead to erroneous results.

The EMD was proposed by Huang et al. [11]. This method decomposes the signal in the time domain into a set of multiple IMFs. These IMFs are AM-FM type of signals. The mathematical equation for EMD method is expressed as,

$$
I M(t)=\sum_{p=1}^{k} U_{p}(t)+S_{k}(t)
$$

where $I M(t)$ presents the original signal in time domain, $U_{p}(t)$ gives the $p^{t h}$ IMF, and $S_{k}(t)$ denotes the residue. Although, this method decomposes the signal into a set of IMFs, it suffers from the mode-mixing problem [14]. Since the method does not account for any apriori about the information for the signal. This gives us different number of IMFs which leads to losing some of the cross channel information present across the channels and even the frequency components are not localized in the frequency domain. To address this issue, a MEMD method has been proposed which simultaneously does the decomposition of all channels. As a result, the number of IMFs obtained remain the same and cross channel information is also utilized. The mathematical expression for MEMD method is given as below:

$$
I T(t)=\frac{1}{p} \sum_{p=1}^{k} e^{\theta_{p}}(t)
$$

where $e^{\theta_{p}}(t)$ gives the envelope curves for multivariate data in all directions vectors and $p$ denotes the length of the vectors. More details may be obtained from [14].

As discussed earlier, these decomposition techniques break the signal into a set of IMFs. Some of the IMFs contain the noise and the residual as well. Hence, this is needed to identify the IMFs which provide the actual information pertaining to any tasks. Henceforward, a filtering method is required to filter the noise out without deterioration of the original signal. Recently, Gaur et.al has proposed a single channel [5] and multichannel filtering [8, 7] technique to handle this issue. These filtering techniques have been built as an enhancement to EMD and MEMD techniques. These filtering techniques first identify the IMF to obtain the reconstructed signal based on signal of interest. They are identified based on the mean frequency measure. Secondly, these identified IMFs are summed up to obtained the enhanced signal. The remaining IMFs are discarded which provide a major contribution to artefacts and noise. 


\section{Results and discussions}

The decomposed components (IMFs) for the EMD based decomposition are shown in Fig. 3. The MEG signals for channel LC21 for multi-direction wrist movements in right, forward, left and backward directions and its obtained first three IMFs are shown. It is clearly evident that the IMFs obtained in this decomposition suffer from localising the frequency components. Also, it has different frequency distribution components present in the same IMFs. If the $\mathrm{IMF}_{5}$ is selected then there may be a strong possibility that we may end up losing some MEG data which actually can help to achieve better feature separability at a later stage.

Figs. 4 and 5 show the obtained MIMFs from MEMD based decomposition method for right hand and forward wrist movements. Three channels MLC32, MLC31, and MLC42 are randomly selected to plot the decomposition mechanism.
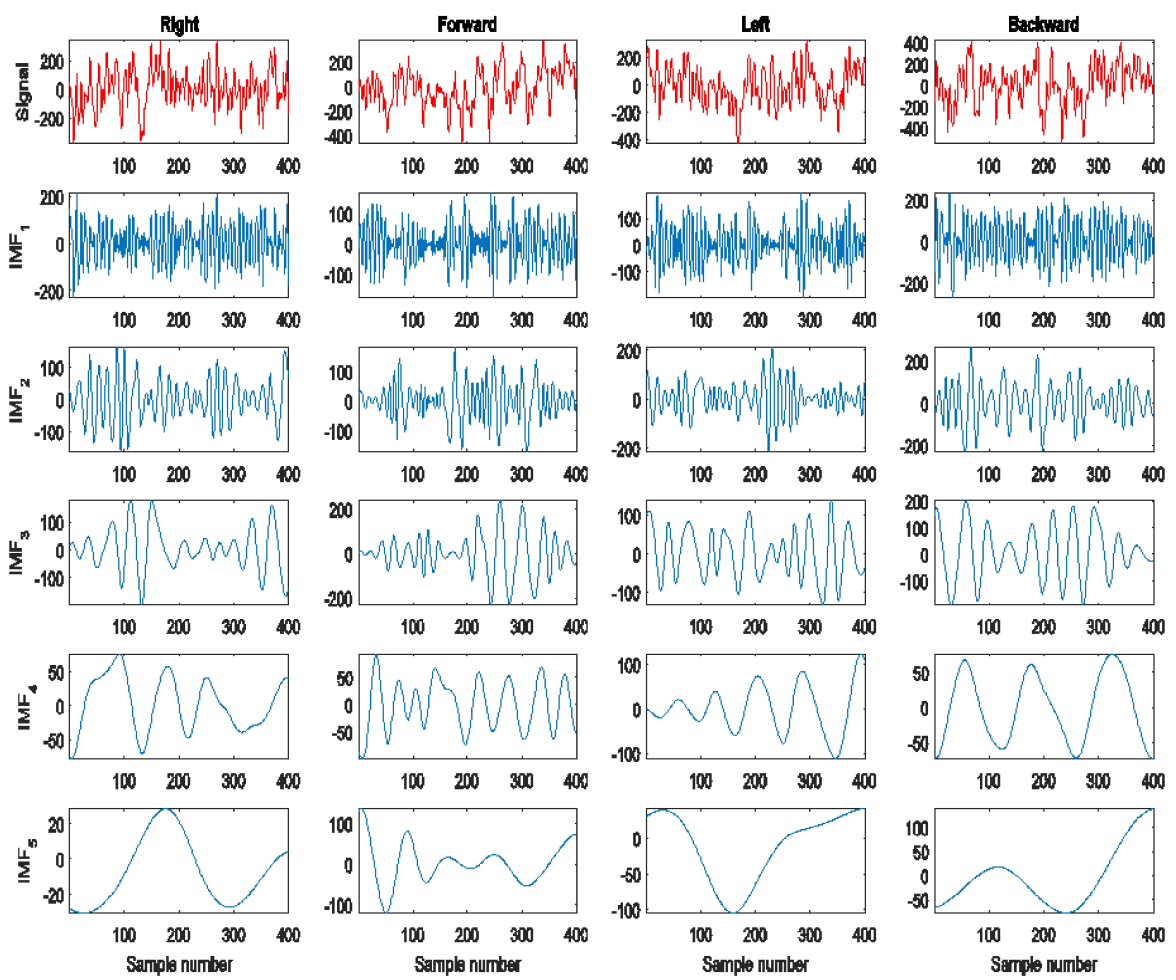

Fig. 3 The MEG signals from channel LC21 for wrist movement to right, forward, left and backward directions and first three IMFs generated.

Although in the actual study all of the ten channels have been considered and decomposed simultaneously. It is clearly evident that the same frequency distribution component is obtained in the same MIMFs as shown in figures 4 and 5 . This 

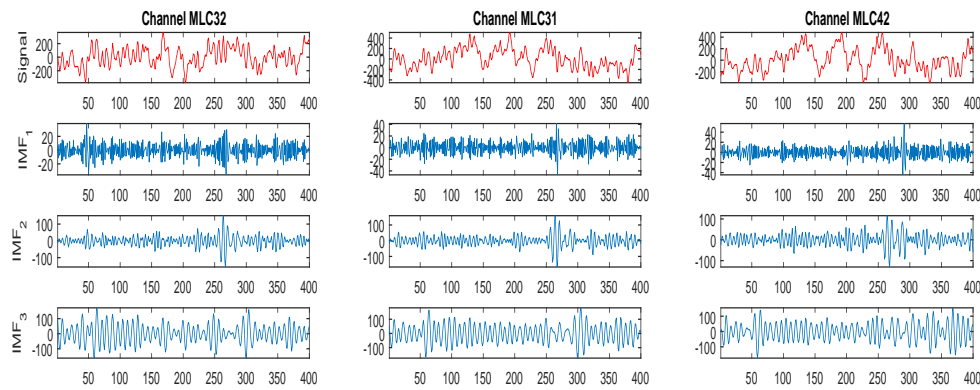

$\begin{array}{llllllllll}50 & 100 & 150 & 200 & 250 & 300 & 350 & 40\end{array}$

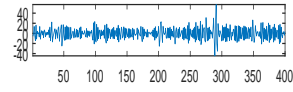

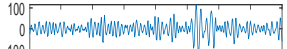

\begin{tabular}{llllllll}
100 & 1 & 1 & 1 & 1 & 1 & 1 & 1 \\
\hline 50 & 100 & 150 & 200 & 250 & 300 & 350 & 400
\end{tabular}

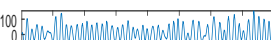

.100

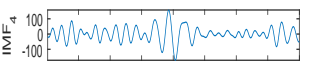

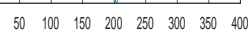

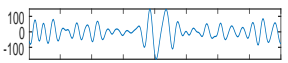

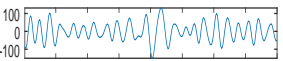

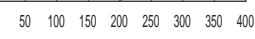

$\stackrel{L_{10}^{50}}{100} \underbrace{}_{50}$

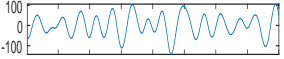

$\begin{array}{llllllll}50 & 100 & 150 & 200 & 250 & 300 & 350 & 40\end{array}$
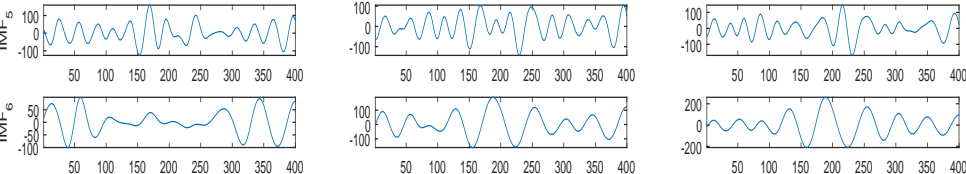

$\begin{array}{llllllll}50 & 100 & 150 & 200 & 250 & 300 & 350 & 400\end{array}$
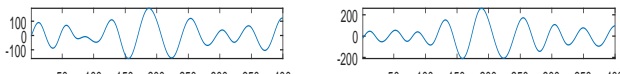

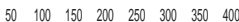
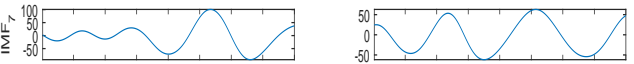

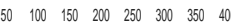

$\begin{array}{llllllll}50 & 100 & 150 & 200 & 250 & 300 & 350 & 400\end{array}$

$30 \quad 100 \quad 150-200 \quad 250 \quad 300 \quad 350 \quad 400$
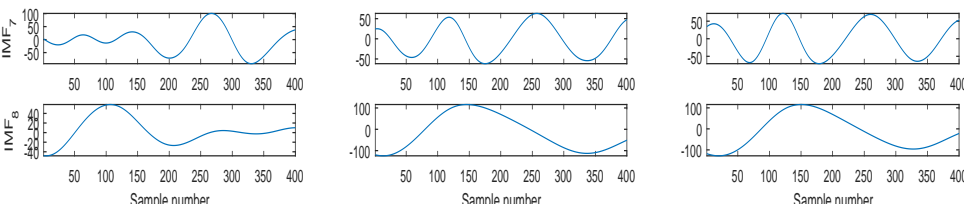

$\begin{array}{llllllll}50 & 100 & 150 & 200 & 250 & 300 & 350 & 400\end{array}$

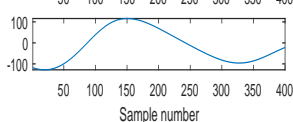

Fig. 4 The MEG signals from three channels for right wrist movement and obtained IMFs.

decomposition helps to utilise the cross-channel information. If a particular MIMFs is/are selected then it is possible to gain high localised frequency without losing information. Similarly, Figs. 6 and 7 display the MIMFs obtained by applying MEMD based decomposition method for left hand and backward wrist movements.

In this work, the shrinkage covariance matrix ( SHCM ) is computed from the enhanced MEG signals obtained from single channel and multi channel filtering methods. Let $F_{1}, F_{2}, F_{3}, \ldots, F_{f}$ denote the $f$ feature vectors. The unbiased estimator of the mean is given as,

$$
\hat{M}=\frac{1}{f} \sum_{i=1}^{f} F_{i}
$$

Also, the unbiased estimator of the covariance matrix is denoted as,

$$
\hat{C}=\frac{1}{f-1} \sum_{i=1}^{f}\left(F_{i}-\hat{M}\right)\left(F_{i}-\hat{M}\right)^{T}
$$

To account for the estimation error, $\hat{C}$ is substituted by 

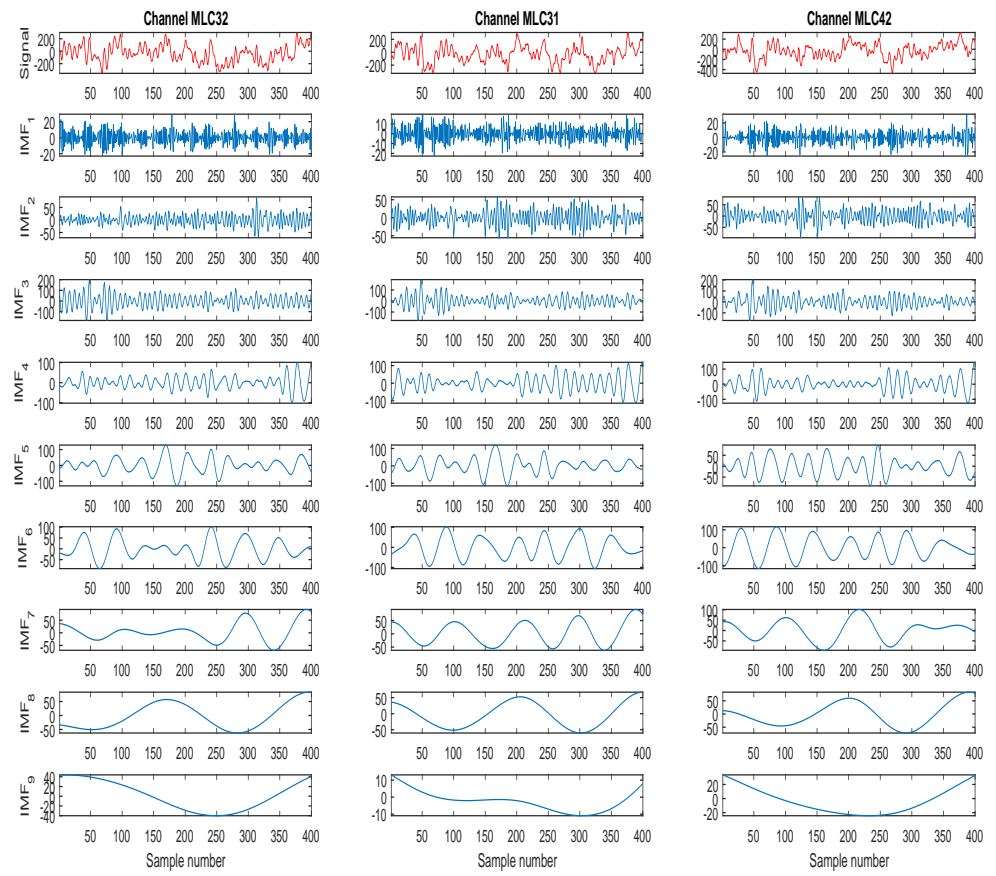

Fig. 5 The MEG signals from three channels for forward wrist movement and obtained IMFs.

$$
C(i)=(1-\gamma) \hat{\sigma}+\gamma v I
$$

with a tuning parameter $(\gamma) \in[0,1] . v$ denotes the average eigenvalue. More details may be obtained from [4].

Table 1 Classification accuracies with the proposed method when evaluated on BCI competition IV dataset 3

\begin{tabular}{ccccccc}
\hline Subject & MEMDBF EMDBF Winner1 & Winner2 & Winner3 & Winner 4 \\
\hline S01 & 52.7 & 40.54 & $\mathbf{5 9 . 5}$ & 31.1 & 16.2 & 23.0 \\
S02 & 49.31 & 43.83 & 34.3 & 19.2 & 31.5 & 17.8 \\
\hline Average & 51.00 & 42.18 & 46.90 & 25.15 & 23.85 & 20.4 \\
Std & 2.4 & 2.33 & 17.82 & 8.41 & 10.82 & 3.68 \\
\hline
\end{tabular}

Table 1 shows the classification accuracy when evaluated on BCI competition IV dataset 3 using EMD based filtering and MEMD based filtering and comparison with BCI competition winners [21]. Here, in Table 1 Std denotes the standard deviation along with classification accuracy comparison with other BCI competition winners. The classification accuracy has been computed for subjects S01 and S02. The main 

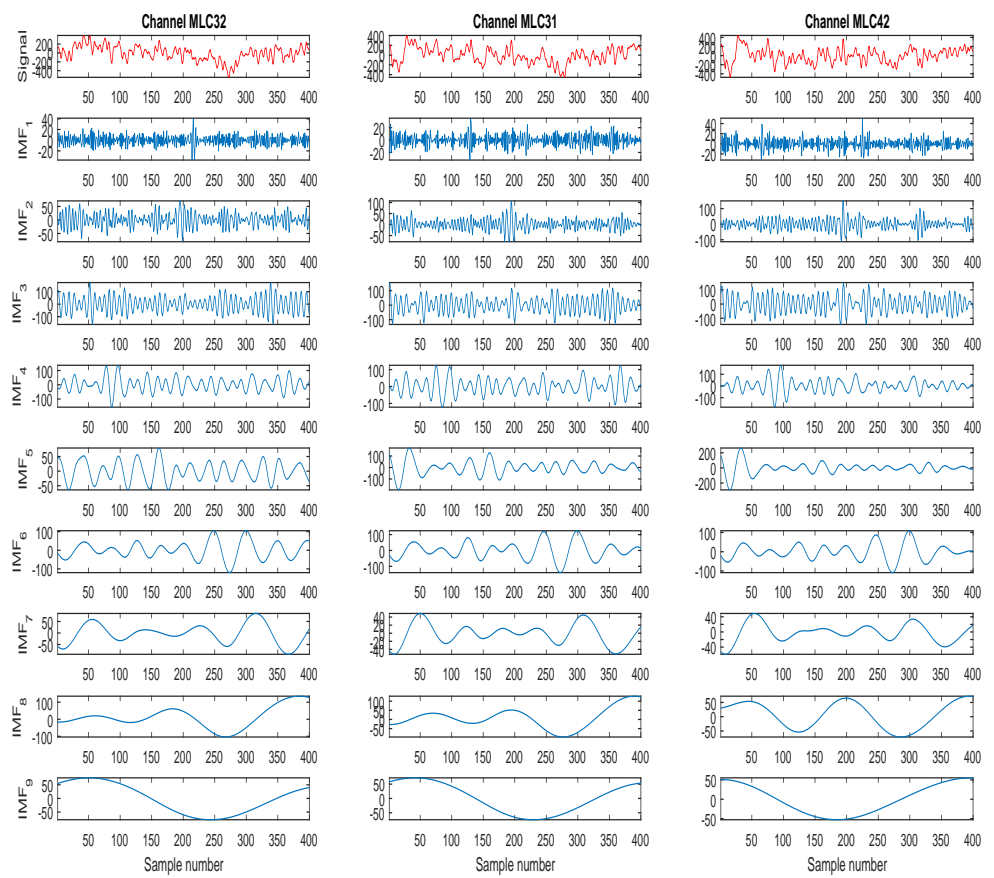

Fig. 6 The MEG signals from three channels for left wrist movement and obtained IMFs.

highlights observed based on the classification accuracy are as follows: (1) The average classification accuracy computed with the EMD based filtering and MEMD based filtering for both subjects gives a minimum standard deviation of 2.33 and 2.4 as compared to BCI competition winners [21]. (2) Subject S02 gives the maximum classification accuracy of $49.31 \%$ which is higher than ( $>5 \%$ ) with EMDBF method and $>15 \%$ with the BCI competition winner [21]. (3) As the higher classification is achieved in the multi-class classification problem using the MEMDBF technique, thus the features are more separable as compared to EMDBF method. These filtering techniques have been served as a preprocessing step. It should be noted that no complexity has been introduced at the feature extraction and classification steps.

Fig 8 shows the graph plot for the comparison of classification accuracies between MEMDBF, EMDBF [6] and BCI competition winners [21]. The improvement obtained in the average classification accuracy across the two subjects is illustrated with the bar graphs. The performance improvement for the BCI dataset IV dataset 3 is shown for the evaluation session. There is a significant improvement in average classification accuracy for subject S02 using the MEMDBF method in the evaluation session. 


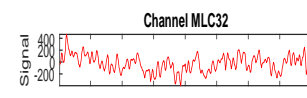

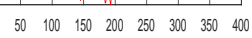

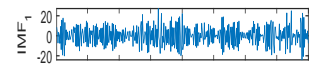

$\begin{array}{llllllll}50 & 100 & 150 & 200 & 250 & 300 & 350 & 400\end{array}$

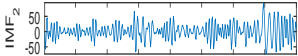

$\begin{array}{llllllll}50 & 100 & 150 & 200 & 250 & 300 & 350 & 400\end{array}$

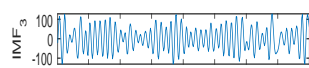

$\begin{array}{llllllll}50 & 100 & 150 & 200 & 250 & 300 & 350 & 400\end{array}$

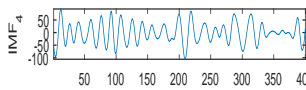

$\stackrel{4}{\Sigma} \quad 50$

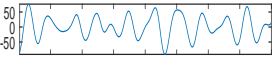

$\begin{array}{llllllll}50 & 100 & 150 & 200 & 250 & 300 & 350 & 400\end{array}$

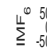

$\begin{array}{llllllll}50 & 100 & 150 & 200 & 250 & 300 & 350 & 400\end{array}$

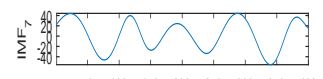

$\begin{array}{llllllll}50 & 100 & 150 & 200 & 250 & 300 & 350 & 400\end{array}$

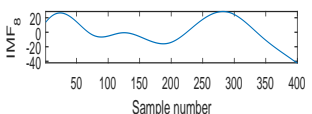

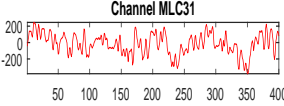

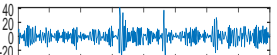

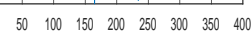

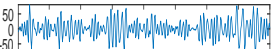

$\begin{array}{llllllll}50 & 100 & 150 & 200 & 250 & 300 & 350 & 400\end{array}$

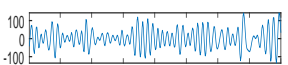

$50 \quad 100 \quad 150 \quad 200 \quad 250 \quad 300 \quad 350 \quad 400$

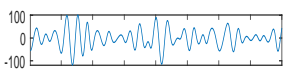

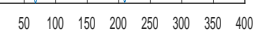

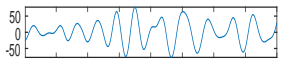

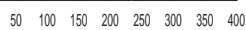

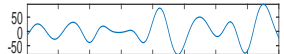

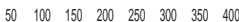
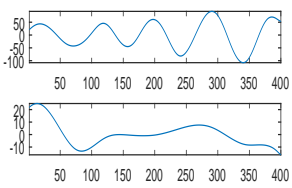

Sample number

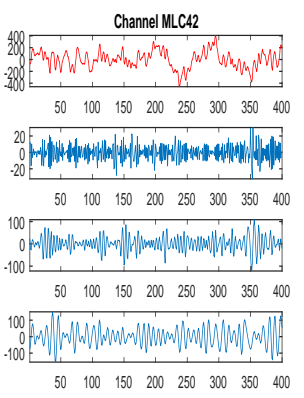

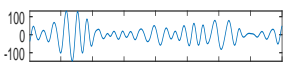

$\begin{array}{llllllll}50 & 100 & 150 & 200 & 250 & 300 & 350 & 400\end{array}$
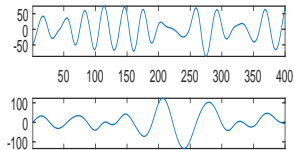

$\begin{array}{llllllll}50 & 100 & 150 & 200 & 250 & 300 & 350 & 400\end{array}$

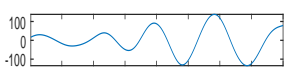

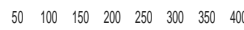

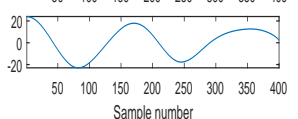

Fig. 7 The MEG signals from three channels for backward wrist movement and obtained IMFs.

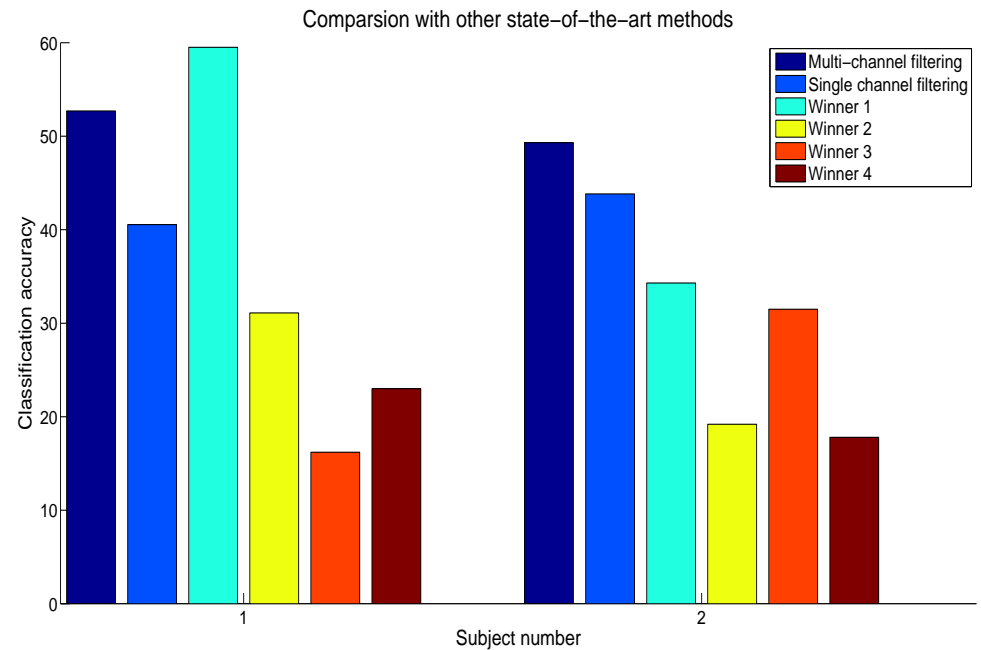

Fig. 8 The bar graphs depict the comparison of classification accuracy with BCI IV competition winners for 2 subjects. The data is taken from BCI Competition IV dataset 3. 


\section{Conclusion}

A comparison analysis of the multi-direction wrist movement MEG signals feature discrimination using the EMD based filtering and MEMD based filtering methods has been undertaken. The shrinkage covariance matrix has been computed as a feature set to classify MEG signals into multiple classes of right, forward, left, and backward movements. These features have been calculated from the enhanced MEG signal obtained from EMD based filtering and MEMD based filtering methods. Consequently, their capability to classify the MEG signals into multiple classes has been explored based on the computed classification accuracy and standard deviation. The minimum standard deviation has been obtained across subjects using these filtering

technique using MEMDBF. Features obtained from MEMDBF provides superior discrimination capability which leads to higher classification accuracy. Thus, using MEMDBF shows good potential in classifying the MEG signals into multiple classes. Future work may involve studying different features such as frequency domain and time-frequency domain features and to employ suitable classifiers, such as support vector machine (SVM) or artificial neural network (ANN) or k-nearest neighbor $(\mathrm{KNN})$ to classify multi-direction wrist movements MEG signals where MEMDBF may be utilized as a preprocessing step. Thus, this study may aid researchers to develop an improved method that would be helpful in rehabilitation for people suffering from strokes.

\section{References}

1. Arvaneh, M., Guan, C., Ang, K.K., Quek, C.: Optimizing spatial filters by minimizing withinclass dissimilarities in electroencephalogram-based brain-computer interface. IEEE Transactions on Neural Networks and Learning Systems 24(4), 610-619 (2013)

2. Barachant, A., Bonnet, S., Congedo, M., Jutten, C.: Multiclass brain-computer interface classification by Riemannian geometry. IEEE Transactions on Biomedical Engineering 59(4), 920-928 (2012)

3. Blankertz, B., Dornhege, G., Krauledat, M., Müller, K.R., Curio, G.: The non-invasive Berlin brain-computer interface: fast acquisition of effective performance in untrained subjects. NeuroImage 37(2), 539-550 (2007)

4. Blankertz, B., Lemm, S., Treder, M., Haufe, S., Müller, K.R.: Single-trial analysis and classification of ERP components - A tutorial. NeuroImage 56(2), 814-825 (2011)

5. Gaur, P., Pachori, R.B., Wang, H., Prasad, G.: An empirical mode decomposition based filtering method for classification of motor-imagery EEG signals for enhancing brain-computer interface. In: International Joint Conference on Neural Networks. pp. 1-7 (2015)

6. Gaur, P., Prasad, G., Wang, H., Pachori, R.: An MEG based BCI for classification of multidirection wrist movements using empirical mode decomposition. MEG UK 2016, York, UK (2016)

7. Gaur, P., Pachori, R.B., Wang, H., Prasad, G.: A multivariate empirical mode decomposition based filtering for subject independent BCI. 27th Irish Signals and Systems Conference (ISSC). pp. 1-7. IEEE (2016)

8. Gaur, P., Pachori, R.B., Wang, H., Prasad, G.: Enhanced Motor Imagery Classification in EEG-BCI using Multivariate EMD based Filtering and CSP Features. Proceedings of the Sixth International Brain-Computer Interface Meeting: BCI Past, Present, and Future (2016) 
9. Gaur, P., Bornot, J., Prasad, G., Wang, H., Pachori, R.: Decoding of Multi-direction Wrist Movements Using Multivariate Empirical Mode Decomposition. In: MEG UK 2017, University of Oxford, UK (2017)

10. Phinyomark, A., Thongpanja, S., Hu, H., Phukpattaranont, P., Limsakul, C.: The usefulness of mean and median frequencies in electromyography analysis. Computational Intelligence in Electromyography Analysis-A Perspective on Current Applications and Future Challenges, InTech, (2012)

11. Huang, N.E., Shen, Z., Long, S.R., Wu, M.C., Shih, H.H., Zheng, Q., Yen, N.C., Tung, C.C., Liu, H.H.: The empirical mode decomposition and the Hilbert spectrum for nonlinear and non-stationary time series analysis. Proceedings of the Royal Society of London A: Mathematical, Physical and Engineering Sciences. vol. 454, pp. 903-995 (1998)

12. Kaushik, G., Gaur, P., Prasad, G., Wang, H., Pachori, R.: An MEG based multi direction wrist movements analysis using empirical mode decomposition and multivariate empirical mode decomposition. MEG UK 2017, University of Oxford, UK (2017)

13. Lotte, F., Guan, C.: Regularizing common spatial patterns to improve BCI designs: unified theory and new algorithms. IEEE Transactions on Biomedical Engineering 58(2), 355-362 (2011)

14. Park, C., Looney, D., Ahrabian, A., Mandic, D.P., et al.: Classification of motor imagery BCI using multivariate empirical mode decomposition. IEEE Transactions on Neural Systems and Rehabilitation Engineering 21(1), 10-22 (2013)

15. Pfurtscheller, G., Neuper, C., Flotzinger, D., Pregenzer, M.: EEG-based discrimination between imagination of right and left hand movement. Electroencephalography and Clinical Neurophysiology 103(6), 642-651 (1997)

16. ur Rehman, N., Park, C., Huang, N.E., Mandic, D.P.: EMD via MEMD: multivariate noiseaided computation of standard EMD. Advances in Adaptive Data Analysis 5(02), 1350007 (2013)

17. Sardouie, S.H., Shamsollahi, M.B.: Selection of efficient features for discrimination of hand movements from MEG using a BCI competition IV data set. Frontiers in Neuroscience, 6 (2012)

18. Tangermann, Michael and Müller, Klaus-Robert and Aertsen, Ad and Birbaumer, Niels and Braun, Christoph and Brunner, Clemens and Leeb, Robert and Mehring, Carsten and Miller, Kai J and Müller-Putz, Gernot R and others.: Review of the BCI Competition IV. Frontiers in Neuroscience, 6 (2012)

19. Sharma, R., Pachori, R.: Automated classification of focal and non-focal EEG signals based on bivariate empirical mode decomposition. Biomedical Signal and Image Processing in Patient Care. IGI Global (2017)

20. Sharma, R., Pachori, R.B.: Classification of epileptic seizures in EEG signals based on phase space representation of intrinsic mode functions. Expert Systems with Applications 42(3), 1106-1117 (2015)

21. Tangermann, M., Müller, K.R., Aertsen, A., Birbaumer, N., Braun, C., Brunner, C., Leeb, R., Mehring, C., Miller, K.J., Müller-Putz, G.R., et al.: Review of the BCI Competition IV. Frontiers in Neuroscience 6 (2012)

22. Wolpaw, J.R., Birbaumer, N., McFarland, D.J., Pfurtscheller, G., Vaughan, T.M.: Braincomputer interfaces for communication and control. Clinical Neurophysiology 113(6), 767791 (2002)

23. Wu, Z., Huang, N.E.: Ensemble empirical mode decomposition: a noise-assisted data analysis method. Advances in Adaptive Data Analysis 1(01), 1-41 (2009) 